\title{
The radiation impedance of electrodynamic tethers in a polar Jovian orbit
}

\author{
A. Sánchez-Torres *, J.R. Sanmartín, J.M. Donoso, M. Charro
}

\begin{abstract}
Juno, the second mission in the NASA New Frontiers Program, will both be a polar Jovian orbiter, and use solar arrays for power, moving away from previous use of radioisotope power systems (RPSs) in spite of the weak solar light reaching Jupiter. The power generation at Jupiter is critical, and a conductive tether could be an alternative source of power. A current-carrying tether orbiting in a magnetized ionosphere/plasmasphere will radiate waves. A magnitude of interest for both power generation and signal emission is the wave impedance. Jupiter has the strongest magnetic field in the Solar Planetary System and its plasma density is low everywhere. This leads to an electron plasma frequency smaller than the electron cyclotron frequency, and a high Alfven velocity. Unlike the low Earth orbit (LEO) case, the electron skin depth and the characteristic size of plasma contactors affect the Alfven impedance.
\end{abstract}

\section{Introduction}

In August 2011 a two-stage Atlas-5(551) is scheduled to launch the first solar-powered mission to Jupiter (Juno). After a deep space maneuver in 2012 to adjust the nominal trajectory and an Earth gravity assist in 2013, the Juno orbiter will reach Jupiter in 2016. The baseline mission consists of 32 highly elliptical polar orbits $\left(i \approx 90^{\circ}, e \approx 0.947\right)$, with a perijove $\sim 1.06 R_{\mathrm{J}}$ and apojove near $39 R_{\mathrm{J}}$. The $J_{2}$ zonal harmonic of the Jovian gravitational field is much greater than at Earth and produces apsidal precession; the Juno apses line lies first in the equatorial plane, but the apojove moves southward over the meridian plane during the mission (Fig. 1). The precession rate for this secular effect is about $1 \%$ orbit. The fall to Jupiter, one year after the first elliptical polar orbit, is planned for planetary protection requirements (Matousek, 2007).

Among several objectives, the Juno mission intends to explore the complex structure of the Jovian polar-magnetosphere and aurora phenomena (Bagenal, 2007). The Io volcanic processes set up acceleration mechanisms along the magnetic flux tube that interact with the Jovian magnetosphere, exhibiting wave coupling and generation of instabilities. The Juno scientific payload includes a plasma/ radio wave experiment capable of detecting low and high frequency waves generated by the Jupiter/Io interaction.

Radioisotope power systems (RPSs) were the power sources used in the past for outer planetary missions. The expensive, man-made plutonium-238 is the source of energy used in fueling these systems. After the Cold War, both USA and Russia have shut down the facilities for these resources. The ${ }^{238} \mathrm{Pu}$ available to NASA is inadequate to support scheduled missions beyond 2018. There is a high-priority recommendation to reestablish production to solve the severe ${ }^{238} \mathrm{Pu}$ demand problem (National Academy of Sciences, 2009). New power generation concepts 


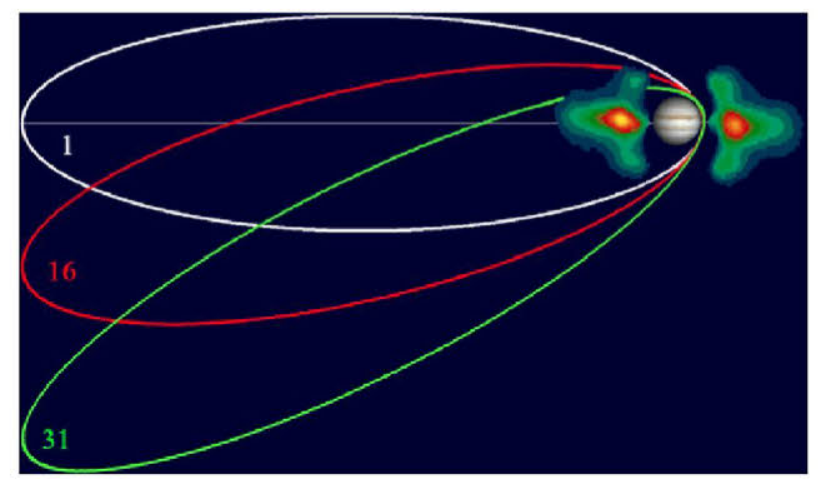

Fig. 1. Elliptical orbits for Juno mission. The influence of Jupiter's oblateness produces apsidal precession over the meridional plane.

may solve this problem. The cancelled JIMO mission would have used nuclear fission energy to reach Jupiter and its moons. Like Juno, the already launched Rosetta spacecraft uses solar arrays for a long-term mission into the main asteroid belt between Mars and Jupiter.

A conductive tether orbiting in the Jovian magnetosphere could be an alternative source of power, the large electromotive force induced driving a current along the tether. Power generation has been considered in two bare-tether missions to the Jovian system, a Jupiter capture operation (Sanmartín et al., 2008) followed by a moon tour (Sanmartín et al., 2009), that uses power generated in the tether, and a Juno-type mission (Bombardelli et al., 2009), proposed to just generate power.

Considerable research has been carried out on the plasma waves radiated by tether systems moving through the Earth's ionosphere. The first analysis to consider this process was carried out by Drell et al. (1965), using the cold plasma approximation, as followed by later authors (Barnett and Olbert, 1986; Dobrowolny and Veltri, 1986; Hastings and Wang, 1987; Estes, 1988; Hastings and Wang, 1989; Donohue et al., 1991). Wave radiation by a tether, however, differs at Juno from the case of equatorial, circular LEO (Sanmartín and Martínez-Sánchez, 1995), because of differences in both geometry and planetary conditions. Whereas orbital/plasma velocities are parallel in circular equatorial orbits, the orbital velocity of Juno lies in the meridional plane, with co-rotation velocity along parallels. Also, plasma density and magnetic field at Jupiter are much smaller and larger, respectively, than in LEO; this results in much higher impedances and modified power spectrum.

In general, there are five branches of the dispersion relation in a cold plasma model, $\omega^{2}(k, \theta)$, with $\theta$ the angle between magnetic field $\mathbf{B}$ and wavenumber $\mathbf{k}$ : fast extraordinary $(\mathrm{FE})$, ordinary $(\mathrm{O})$, slow extraordinary $(\mathrm{SE})$, fast magnetosonic or compressional Alfven (FMS), and Alfven or shear Alfven (A). In our case, as in LEO, the refraction index is very large in all cases $\left(c \gg V_{\text {rel }}\right)$,

$n \equiv \frac{c k}{\omega}=\frac{c k}{\mathbf{V}_{\text {rel }} \cdot \mathbf{k}} \gg 1$ where $\mathbf{V}_{\text {rel }}=\mathbf{V}_{\text {orb }}-\mathbf{V}_{\text {pl }}$ is the tether velocity relative to the co-rotating plasma and $c$ is the velocity of light. Only slow extraordinary, fast magnetosonic, and Alfven waves allow $n>1$ values and thus steady current emission in a cold plasma model. This model can be used only if the phase velocity of the emitted waves is faster than the thermal speed of the particles. In the following our study will focus on Alfven waves. For a tether carrying a quasi-steady current, the wave frequency in the plasma frame is given by the Doppler relation

$\omega=\mathbf{V}_{\text {rel }} \cdot \mathbf{k} \simeq \mathbf{V}_{\text {rel } \perp} \cdot \mathbf{k}_{\perp}$

where, as we shall later see, $\mathbf{k}$ is nearly perpendicular to the ambient magnetic field.

\section{Ambient and orbital Juno-like conditions}

Following a model of the magnetosphere of Jupiter (Divine and Garrett, 1983), the plasma velocity, along parallels, is, from the rotation period in Table 1

$V_{\mathrm{pl}}=12.6 \frac{r_{\perp}}{R_{\mathrm{J}}} \frac{\mathrm{km}}{\mathrm{s}}, \quad r_{\perp}=r \cos \lambda$

where $\lambda$ is both latitude at Jupiter and true anomaly in the first orbit. Due to the high eccentricity, the orbit can be approximated as parabolic near Jupiter,

$1+\cos \lambda \simeq \frac{2 r_{\mathrm{p}}}{r}$

where $r_{\mathrm{p}}$ is the perijove distance. The orbital velocity $V_{\text {orb }}$, in the meridian plane, then reads

$V_{\text {orb }} \simeq \sqrt{\frac{2 \mu_{\mathrm{J}}}{r}} \approx 59.7 \sqrt{\frac{R_{\mathrm{J}}}{r}} \frac{\mathrm{km}}{\mathrm{s}}$

The relative velocity $V_{\text {rel }}$ itself is very close to $V_{\text {orb }}$,

$V_{\text {rel }} \simeq V_{\text {orb }}\left[1+\frac{1}{2}\left(\frac{12.6}{59.7}\right)^{2}\left(\frac{r}{R_{\mathrm{J}}}\right)^{3} \cos ^{2} \lambda\right]$

Note that the orbital velocity is different from zero everywhere, whereas the plasma velocity vanishes at $\lambda=\pi / 2$.

Regarding the magnetic field, we ignore the tilt in the magnetic-dipole approximation and consider the following scalar potential

$\psi=-\frac{\mu_{0} B_{0} R_{\mathrm{J}}^{3}}{4 \pi} \frac{\sin \lambda}{r^{2}}$

Table 1

Characteristic values of Earth and Jupiter parameters.

\begin{tabular}{|c|c|c|c|}
\hline Parameters & Jupiter & Earth & Ratio (Jupiter/Earth) \\
\hline Equatorial radius $(\mathrm{km})$ & 71,492 & 6378 & 11.2 \\
\hline$\mu=G M\left(\mathrm{~km}^{3} / \mathrm{s}^{2}\right)$ & 126.6 & 0.4 & 316.5 \\
\hline$J_{2}\left(10^{-6}\right)$ & 14,736 & 1082.6 & 13.6 \\
\hline Rotation period & $9 \mathrm{~h} 55 \mathrm{~min}$ & $23 \mathrm{~h} 56 \mathrm{~min}$ & 0.41 \\
\hline $\begin{array}{l}\text { Pasmasphere/ionosphere } \\
\text { species }\end{array}$ & $\mathrm{S}^{+}, \mathrm{O}^{+}$ & $\mathrm{O}^{+}, \mathrm{H}^{+}, \mathrm{He}^{+}$ & - \\
\hline
\end{tabular}


where $B_{0} \approx 4.23$ gauss is the surface magnetic field at the equator. The magnetic field modulus itself reads

$B=B_{0}\left(\frac{R_{J}}{r}\right)^{3} \sqrt{1+3 \sin ^{2} \lambda}$

The thermal plasma density, from the Divine Garrett model for the inner plasmasphere $\left(1.0<r<3.8 R_{\mathrm{J}}\right)$, is

$N_{\mathrm{e}}=4.65 \times \exp \left[7.68 \frac{R_{\mathrm{J}}}{r}-\left(\frac{r}{R_{\mathrm{J}}}-1\right)^{2} \lambda^{2}\right] \mathrm{cm}^{-3}$

Considering cyclotron and plasma frequencies for each species

$\Omega_{\sigma}=\frac{e B}{m_{\sigma}}, \quad \omega_{p \sigma}=\left(\frac{N_{\mathrm{e}} e^{2}}{m_{\sigma} \varepsilon_{0}}\right)^{1 / 2} \quad$ with $\sigma=e, i$

and assuming sulphur ions as prevailing species, the Alfven velocity can be expressed as

$V_{\mathrm{A}}=c \frac{\Omega_{i}}{\omega_{\mathrm{pi}}} \approx 3.85 \times 10^{5} \frac{B \text { [gauss] }}{\sqrt{N_{\mathrm{e}}\left[\mathrm{cm}^{-3}\right]}} \frac{\mathrm{km}}{\mathrm{s}}$

The Alfven velocity in Eq. (11) is large as compared to the LEO case because of the high and low values in Eqs. (8) and (9), respectively. This should result in a large Alfven impedance. Also, near $\lambda=\pi / 2$, where $V_{\mathrm{A}}$ approaches $c$, the lower hybrid frequency will read

$\omega_{\mathrm{LH}} \simeq \sqrt{\omega_{\mathrm{pi}}^{2}+\Omega_{i}^{2}} \simeq \omega_{\mathrm{pi}} \sqrt{1+\frac{V_{\mathrm{A}}^{2}}{c^{2}}}$

whereas $\omega_{\mathrm{LH}} \simeq \omega_{\mathrm{pi}}$ holds in LEO. Finally, at Jupiter we have

$V_{\mathrm{A}}^{2} \gg \frac{m_{i}}{m_{\mathrm{e}}} V_{\mathrm{rel} \perp}^{2}$

whereas the opposite applies in LEO. Eq. (13) can be rewritten as

$\frac{c^{2}}{\omega_{\mathrm{pe}}^{2}} \gg \frac{V_{\mathrm{rel} \perp}^{2}}{\Omega_{i}^{2}}$

This will result in a different Alfven power spectrum.

\section{The wave field from a current-density tether source}

Using the equation for the Fourier transform of the electric field $\mathbf{E}$ of a wave, we have (Akhiezer et al., 1975)

$-\frac{\mathbf{k} \wedge(\mathbf{k} \wedge \mathbf{E})}{k^{2}}-\frac{\overline{\bar{\varepsilon}}_{\mathrm{c}} \cdot \mathbf{E}}{n^{2}}=\frac{4 \pi i \mathbf{j}_{\mathrm{s}}}{\omega n^{2}}$

where $\mathbf{j}_{\mathrm{s}}$ is the current source and where the dielectric tensor of a cold plasma reads

$\overline{\bar{\varepsilon}}_{\mathrm{c}}(\omega)=\left(\begin{array}{ccc}\varepsilon_{1} & i \varepsilon_{2} & 0 \\ -i \varepsilon_{2} & \varepsilon_{1} & 0 \\ 0 & 0 & \varepsilon_{3}\end{array}\right)$ with the $z$-axis taken along the Jovian magnetic field $\mathbf{B}$ at each local position. The Astrom dispersion relation, for nontrivial solutions of Eq. (15) with $\mathbf{j}_{\mathrm{s}}=0$, reads

$$
\begin{aligned}
\mathscr{D}(k, \theta, \omega) \equiv & \left(\varepsilon_{1} \sin ^{2} \theta+\varepsilon_{3} \cos ^{2} \theta-\frac{\varepsilon_{3} \varepsilon_{1}}{n^{2}}\right)\left(1-\frac{\varepsilon_{1}}{n^{2}}\right) \\
& +\left(\sin ^{2} \theta-\frac{\varepsilon_{3}}{n^{2}}\right) \frac{\varepsilon_{2}^{2}}{n^{2}}=0
\end{aligned}
$$

where $\theta$ is the angle between $\mathbf{B}$ and $\mathbf{k}$. Introducing the longitudinal and transverse parts of the electric field (Sanmartín and Martínez-Sánchez, 1995),

$\mathbf{E}_{l} \equiv \mathbf{k} \mathbf{k} \cdot \frac{\mathbf{E}}{k^{2}}=-i \mathbf{k} \phi, \quad \mathbf{E}_{\mathrm{t}}=\mathbf{E}-\mathbf{E}_{l}$

solving for $\mathbf{E}_{\mathrm{t}}\left(\mathbf{E}_{l}, \mathbf{j}_{\mathrm{s}}\right)$, and using $\mathbf{k} \cdot \mathbf{E}_{\mathrm{t}}=0$, finally yields

$$
\begin{aligned}
& k^{2} \mathscr{D}(k, \theta, \omega) \phi= {\left[\left(1-\frac{\varepsilon_{1}}{n^{2}}\right)^{2}-\frac{\varepsilon_{2}^{2}}{n^{4}}\right] \frac{4 \pi}{\omega}\left[k_{z} j_{s z}\right.} \\
&\left.+\left(1-\frac{\varepsilon_{3}}{n^{2}}\right) \mathbf{k}_{\perp} \cdot \mathscr{H}^{-1} \cdot \mathbf{j}_{s \perp}\right] \\
& \mathscr{H}=\mathscr{I}-\frac{1}{n^{2}}\left(\begin{array}{cc}
\varepsilon_{1} & i \varepsilon_{2} \\
-i \varepsilon_{2} & \varepsilon_{1}
\end{array}\right)
\end{aligned}
$$

where $\mathscr{I}$ is the two-dimensional unit tensor.

For Alfven waves $\left(\omega<\Omega_{i}\right)$, the matrix elements of the dielectric tensor are simplified

$\varepsilon_{1} \approx \frac{\omega_{\mathrm{pi}}^{2}+\Omega_{i}^{2}-\omega^{2}}{\Omega_{i}^{2}-\omega^{2}}$,
$\frac{\varepsilon_{1}}{n^{2}}=\frac{\Omega_{i}^{2}\left(1+c^{2} / V_{\mathrm{A}}^{2}-\omega^{2} / \Omega_{i}^{2}\right)}{\Omega_{i}^{2}-\omega^{2}} \cdot \frac{V_{\mathrm{rel} \perp}^{2} k_{\perp}^{2}}{c^{2} k^{2}} \ll 1$

$\frac{\varepsilon_{2}}{n^{2}} \approx \frac{\omega}{\Omega_{i}} \frac{\varepsilon_{1}}{n^{2}} \ll 1$

$-\frac{\varepsilon_{3}}{n^{2}} \approx \frac{\omega_{\mathrm{pe}}^{2}}{c^{2} k^{2}}=\frac{m_{i} V_{\mathrm{rel} \perp}^{2} \Omega_{i}^{2} k_{\perp}^{2}}{m_{\mathrm{e}} V_{\mathrm{A}}^{2} \omega^{2} k^{2}} \leqslant \mathscr{O}(1)$

As in LEO, we have $\varepsilon_{1} / n^{2}, \varepsilon_{2} / n^{2}$ small and $\mathscr{H} \simeq \mathscr{I}$, whereas $-\varepsilon_{3} / n^{2}$ was large in LEO, however. The dispersion relation expressed in Eq. (17) is now approximated as

$\mathscr{D} \approx \mathscr{D}_{\mathrm{J}}(k, \theta, \omega)=\varepsilon_{1}\left(\sin ^{2} \theta-\frac{\varepsilon_{3}}{n^{2}}\right)+\varepsilon_{3} \cos ^{2} \theta$,

$\cos \theta \equiv \frac{k_{z}}{k} \ll 1$

The tether, which is assumed rotating in the orbital plane to keep it taut in the presence of the weak Jovian gravity gradient, will be at anytime at some angle $\varphi(t)$ with the magnetic field (Fig. 2). Since the $z$ component of the wave vector is small, the Doppler relation reads

$\omega \approx \mathbf{k}_{\perp} \cdot\left(\mathbf{V}_{\text {orb }}-\mathbf{V}_{\mathrm{pl}}\right) \simeq k_{y} V_{\mathrm{orb}_{y}}-k_{x} V_{\mathrm{pl}} \equiv \omega\left(\mathbf{k}_{\perp}\right)$

where we take the $y$-axis in the orbital polar plane, like both tether and magnetic field, with the co-rotating plasma velocity $V_{\text {pl }}$ then along the $x$-axis (see Fig. 2).

Using Eqs. (15) and (18) the transverse part of the electric field is shown negligible, $\mathbf{E}_{\mathrm{t}} \approx \mathcal{O}\left(n^{-2}\right)$, and the electric 


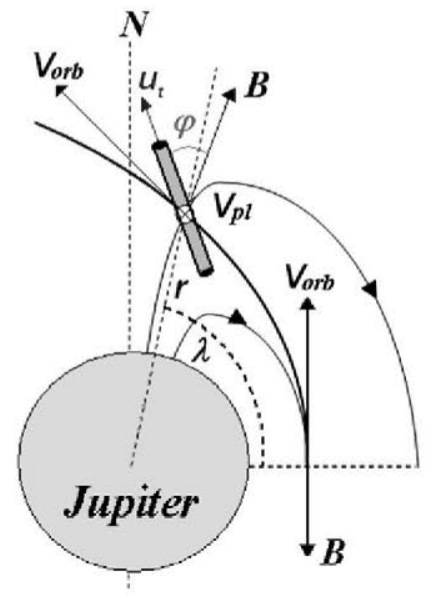

Fig. 2. Coordinate reference system used to compute the tether impedance. We can see the parabolic orbit near Jupiter and its magnetic field configuration. $\mathbf{u}_{\mathrm{t}}$ is the direction along the tether for each rotation angle $\varphi$. At $\lambda=0$ the magnetic field $\mathbf{B}$ is antiparallel to the orbital velocity $\mathbf{V}_{\text {orb }}$. At $\lambda=\pi / 2$ the plasma velocity $\mathbf{V}_{\mathrm{pl}}=0$, and the orbital velocity $\mathbf{V}_{\text {orb }}$ lies at $\pi / 4$ from the magnetic field $\mathbf{B}$.

field is therefore both near longitudinal and near perpendicular to the magnetic field,

$\mathbf{E} \approx-i \mathbf{k}_{\perp} \phi$

with the electric potential

$\phi \simeq \frac{4 \pi}{\omega} \mathbf{k} \cdot \mathbf{j}_{\mathrm{s}} F, \quad F=\frac{1-\varepsilon_{3} / n^{2}}{k^{2} \mathscr{D}}$

where $\mathscr{D}$ is given by Eq. (24).

\section{The radiation impedance formula}

Since the source current vanishes outside certain volume, the power radiated can be written as (Sanmartín and Martínez-Sánchez, 1995)

$$
\begin{aligned}
\dot{W}_{\text {rad }}= & -\int \mathbf{j}_{\mathrm{s}} \cdot \mathbf{E} d \mathbf{r}=-\int \phi \nabla \cdot \mathbf{j}_{\mathrm{s}} d \mathbf{r} \\
= & -\int d \mathbf{r} \int \frac{d \mathbf{k}_{1} d \omega_{1}}{4 \pi^{2}} e^{i \mathbf{k}_{1} \cdot \mathbf{r}-i \omega_{1} t} \phi\left(\mathbf{k}_{1}, \omega_{1}\right) \\
& \times \int \frac{d \mathbf{k} d \omega}{4 \pi^{2}} e^{i \mathbf{k} \cdot \mathbf{r}-i \omega t} i \mathbf{k} \cdot \mathbf{j}_{\mathrm{s}}(\mathbf{k}, \omega)
\end{aligned}
$$

The $\mathbf{r}$ integral is $\int d \mathbf{r} \exp \left[i\left(\mathbf{k}_{1}+\mathbf{k}\right) \cdot \mathbf{r}\right]=8 \pi^{3} \delta\left(\mathbf{k}_{1}+\mathbf{k}\right)$, making the $\mathbf{k}_{1}$ integration inmediate. The general source divergence can be rewritten as:

$$
\begin{aligned}
i \mathbf{k} \cdot \mathbf{j}_{\mathrm{s}} & =\int \frac{d \mathbf{r} d t}{4 \pi^{2}} e^{-i \mathbf{k} \cdot \mathbf{r}+i \omega t} \nabla \cdot \mathbf{j}_{\mathrm{s}}\left(x+V_{\mathrm{pl}} t, y-V_{\mathrm{orb}, y} t, z\right) \\
& \equiv i I_{\mathrm{s}} \delta\left(\omega-\mathbf{V}_{\text {rel }} \cdot \mathbf{k}\right) g(\mathbf{k})
\end{aligned}
$$

where we set $x \rightarrow x-V_{\mathrm{pl}} t, y \rightarrow y+V_{\mathrm{orb}, y} t$ and introduced the current $I_{\mathrm{s}}$ in the tether and a dimensionless function $g(\mathbf{k})$ defined by

$$
g(\mathbf{k})=-i \int d \mathbf{r} \nabla \cdot j_{\mathrm{s}}(\mathbf{r}) e^{-i \mathbf{k} \cdot \mathbf{r}} / 2 \pi I_{\mathrm{s}}
$$

With $\phi \propto \mathbf{k} \cdot \mathbf{j}_{\mathrm{s}}$ in Eq. (27), both $\omega$ and $\omega_{1}$ integrations are inmediate too.

Eq. (28) then reads

$\dot{W} / I_{\mathrm{s}}^{2}=Z_{A \varphi} \equiv \int \frac{2 i|g|^{2} F}{\omega} d \mathbf{k}$

where $Z_{A \varphi}$ is a (current independent) impedance later found to depend on angle $\varphi$ in Fig. 2. Using Barker's equation, the time $\Delta t=8 r_{\mathrm{p}} / 3 V_{\mathrm{orb}}(\lambda=0) \approx 1 \mathrm{~h}$ to move from $\lambda=0$ to $\lambda=\pi / 2$ in the parabolic orbit, is reasonably large compared with a typical spin period $\sim 10 \mathrm{~min}$. We can then find a representative impedance by averaging over one tether revolution at given orbital position

$Z_{\mathrm{A}}=\int_{0}^{2 \pi} \frac{d \varphi}{2 \pi} Z_{A \varphi}=\frac{\dot{W}_{\mathrm{rad}}}{I_{\mathrm{s}}^{2}}=\int_{0}^{2 \pi} \frac{d \varphi}{2 \pi} \int \frac{2 i|g(k)|^{2} d \mathbf{k}}{\omega} F$

where

$F=\frac{1+\left(\omega_{\mathrm{pe}} / c k\right)^{2}}{\varepsilon_{3}\left\{k_{z}^{2}+k_{\perp}^{2}\left[1+\left(\omega_{\mathrm{pe}} / c k\right)^{2}\right] \varepsilon_{1} / \varepsilon_{3}\right\}}$

with $k^{2} \simeq k_{\perp}^{2}$. Actually, $Z_{A \varphi}$ will be found to depend very weakly on $\varphi$. If we make $\omega_{\mathrm{pe}}^{2} \gg c^{2} k_{\perp}^{2}$ and $V_{\mathrm{A}}^{2} \ll c^{2}$ in Eqs. (32) and (33), with no tether spin averaging, we would recover the impedance in the equation (13) of Sanmartín and Martínez-Sánchez (1995). The impedance here is now rewritten as

$$
\begin{aligned}
Z_{\mathrm{A}}= & \int_{0}^{2 \pi} \frac{d \varphi}{2 \pi} \int \frac{2 i|g(k)|^{2} d \mathbf{k}_{\perp}}{\omega} \frac{-\omega^{2}}{\omega_{\mathrm{pe}}^{2}}\left(1+\frac{\omega_{\mathrm{pe}}^{2}}{c^{2} k_{\perp}^{2}}\right) \\
& \times \int_{-\infty}^{\infty} d k_{z}\left\{\left[k_{z}^{2}-k_{\perp}^{2} \frac{\omega^{2} \varepsilon_{1}}{\omega_{\mathrm{pe}}^{2}}\left(1+\frac{\omega_{\mathrm{pe}}^{2}}{c^{2} k_{\perp}^{2}}\right)\right]\right\}^{-1}
\end{aligned}
$$

and evaluating the integral over the $k_{z}$ poles, with $\omega \rightarrow \omega+i v\left(v \rightarrow 0^{+}\right)$, we find

$$
Z_{\mathrm{A}}=\frac{2 \pi}{\omega_{\mathrm{pe}}} \int_{0}^{2 \pi} \frac{d \varphi}{2 \pi} \int \frac{|g|^{2} d \mathbf{k}_{\perp}}{k_{\perp} \sqrt{\varepsilon_{1}}} \sqrt{1+\frac{\omega_{\mathrm{pe}}^{2}}{c^{2} k_{\perp}^{2}}}
$$

Eq. (35) finally reading

$$
\begin{aligned}
Z_{\mathrm{A}}= & \frac{2 \pi V_{\mathrm{A}}}{c^{2} \sqrt{1+\left(V_{\mathrm{A}} / c\right)^{2}}} \int_{0}^{2 \pi} \frac{d \varphi}{2 \pi} \int \frac{|g|^{2} d \mathbf{k}_{\perp}}{k_{\perp}^{2}} \\
& \times \frac{\sqrt{1-\omega^{2} / \Omega_{i}^{2}} \sqrt{1+c^{2} k_{\perp}^{2} / \omega_{\mathrm{pe}}^{2}}}{\sqrt{1-\widetilde{V}_{\mathrm{A}}^{2} \omega^{2} / \Omega_{i}^{2}}}
\end{aligned}
$$

where $\omega$ is the frequency given by Eq. (25), and $\widetilde{V}_{\mathrm{A}}^{2} \equiv V_{\mathrm{A}}^{2} /\left(V_{\mathrm{A}}^{2}+c^{2}\right)$.

To model the current divergence $\nabla \cdot \mathbf{j}_{s}(\mathbf{r})$, and thus $g(\mathbf{k})$, we assume, as generally assumed in the literature, that it occurs on surfaces at the tether ends of dimension small compared with $L$. The simplest, generic model uses a spherical surface, yielding (Sanmartín and Martínez-Sánchez, 1995) 


$$
\nabla \cdot \mathbf{j}_{\mathrm{s}}(\mathbf{r})=\frac{I_{\mathrm{s}}}{4 \pi R^{2}}\left[\delta\left(\left|\frac{L}{2} \mathbf{u}_{\mathrm{t}}+\mathbf{r}\right|-R\right)-\delta\left(\left|-\frac{L}{2} \mathbf{u}_{\mathrm{t}}+\mathbf{r}\right|-R\right)\right]
$$

where $R \ll L$ and $\mathbf{r}$ goes from each tether end to points on its respective surface. For an upward current and a tether length $L$, Eqs. (30) and (37) give

$$
g(\mathbf{k}) \simeq \frac{1}{\pi} \sin \left(\frac{L}{2} k_{y} \sin \varphi\right) \frac{\sin (k R)}{k R}
$$

with $\mathbf{k} \cdot \mathbf{u}_{\mathrm{t}}=k_{z} \cos \varphi+k_{y} \sin \varphi \simeq k_{y} \sin \varphi$ and $\mathbf{u}_{\mathrm{t}}$ the unit vector along the tether for each rotation angle $\varphi$ in Fig. 2.

It was suggested by Donohue et al. (1991), Estes (1988), and Sanmartín and Martínez-Sánchez (1995) that nonlinear effects would make $I_{\mathrm{s}}$ be the random current to the effective contactor surface

$4 \pi R^{2} j_{\text {th }}=I_{\mathrm{s}}$

where $j_{\text {th }} \equiv e N_{\mathrm{e}}\left(k_{B} T_{\mathrm{e}} / 2 \pi m_{\mathrm{e}}\right)^{1 / 2}$ is the random current density. To just estimate the correspondence between "contactor radius" $R$ and current $I_{\mathrm{s}}$, we use $j_{\text {th }}$ from the DivineGarrett model at $1.09 R_{\mathrm{J}}$ on the equator; for $R=0.5 \mathrm{~m}$ and $4 \mathrm{~m}$, we find $I_{\mathrm{s}}=3 \mathrm{~mA}$ and $195 \mathrm{~mA}$, respectively.

Note that Eq. (36) is not separately even on $k_{x}$ and $k_{y}$, except at $\lambda=0$, where $V_{\mathrm{orb}_{y}}=0$ and $\omega=-V_{\mathrm{p} 1} k_{x}$, and $\lambda=\pi / 2$, where $V_{\mathrm{pl}}=0$ and $\omega=k_{y} V_{\mathrm{orb}} / \sqrt{2}$. We focus the study in these two simple extreme cases, with the $\mathbf{k}_{\perp}$ integral then expressed as $\int d k_{x} \int d k_{y}=4 \int_{\geqslant 0} d k_{x} \int_{\geqslant 0} d k_{y}$.

\section{The radiation impedance at the equator}

In the equatorial case the $k_{x}$ wave vector is limited to values $k_{x} \leqslant k_{\mathrm{M}}$, and we have $V_{\mathrm{A}}^{2} \ll c^{2}$. The impedance in Eq. (36) can now written as

$Z_{\mathrm{A}}=\frac{8 V_{\mathrm{A}}}{\pi c^{2}} \times \int_{0}^{\pi / 2} \frac{d \varphi}{\pi / 2} \mathscr{Z}_{\mathrm{A}}$

with

$$
\begin{aligned}
\mathscr{Z}_{\mathrm{A}} \simeq & \int_{0}^{k_{\mathrm{M}}} d k_{x} \sqrt{1-\frac{k_{x}^{2}}{k_{\mathrm{M}}^{2}}} \int_{0}^{\infty} \frac{d k_{y}}{k_{\perp}^{2}} \\
& \times \sin ^{2}\left(k_{y} \frac{L}{2} \sin \varphi\right) \sqrt{1+\frac{k_{\perp}^{2}}{k_{m}^{2}}} \frac{\sin ^{2}\left(k_{\perp} / k_{\mathrm{R}}\right)}{\left(k_{\perp} / k_{\mathrm{R}}\right)^{2}}
\end{aligned}
$$

where

$k_{m} \equiv \frac{\omega_{\mathrm{pe}}}{c} \sim \frac{1}{73.2 \mathrm{~m}}, \quad k_{\mathrm{M}} \equiv \frac{\Omega_{i}}{V_{\mathrm{pl}}} \sim \frac{1}{14.2 \mathrm{~m}}$,

$k_{\mathrm{R}} \equiv \frac{1}{R} \sim \frac{1}{2 \mathrm{~m}}$

with the following ordering

$\frac{1}{L} \ll k_{m} \ll k_{\mathrm{M}} \ll k_{\mathrm{R}}$

In Eq. (41), the $k_{x}$ integrand can be divided into two regions
1. Region $1\left(\mathscr{Z}_{A 1}\right): k_{\text {int }}<k_{x} \leqslant k_{\mathrm{M}}$.

2. Region $2\left(\mathscr{Z}_{A 2}\right): 0 \leqslant k_{x} \leqslant k_{\text {int }}$

with the intermediate value $k_{\text {int }}$ satisfying $1 / L \ll k_{\text {int }} \ll k_{m}$.

For the first region, changing the integration from $k_{y}$ to $k_{\perp} \equiv \sqrt{k_{x}^{2}+k_{y}^{2}}$, at fixed $k_{x}, \mathscr{Z}_{A 1}$ reads

$\mathscr{Z}_{A 1} \simeq \int_{k_{\mathrm{int}}}^{k_{\mathrm{M}}} d k_{x} \sqrt{1-\frac{k_{x}^{2}}{k_{\mathrm{M}}^{2}} I}$

where

$I=\int_{k_{x}}^{\infty} \frac{d k_{\perp}}{2 k_{\perp}} \frac{\sqrt{1+k_{\perp}^{2} / k_{m}^{2}}}{\sqrt{k_{\perp}^{2}-k_{x}^{2}}} \frac{\sin ^{2}\left(k_{\perp} / k_{\mathrm{R}}\right)}{\left(k_{\perp} / k_{\mathrm{R}}\right)^{2}}$

and we set $\sin ^{2}\left(\frac{1}{2} k_{y} L \sin \varphi\right) \approx 1 / 2$. Introducing $k_{\perp}^{*}$, such that $k_{\text {int }} \leqslant k_{x} \leqslant k_{\mathrm{M}} \ll k_{\perp}^{*} \ll k_{\mathrm{R}}$, the $I$ integral can itself be divided into two parts (see Fig. 3):

(a) $I_{a}: k_{x} \leqslant k_{\perp}<k_{\perp}^{*}$. We can here use $\sin ^{2}\left(k_{\perp} / k_{\mathrm{R}}\right) /$ $\left(k_{\perp} / k_{\mathrm{R}}\right)^{2} \approx 1$.

(b) $I_{b}: k_{\perp}^{*}<k_{\perp}<\infty$. In this broad range, we use the approximations $k_{\perp} \gg k_{x}$ and $k_{\perp} \gg k_{m}$.

For integrals $I_{a}$ and $I_{b}$, we readily find

$$
\begin{aligned}
I_{a} & \simeq \int_{k_{x}}^{k_{\perp}^{*}} \frac{d k_{\perp}}{2 k_{\perp}} \frac{\sqrt{1+k_{\perp}^{2} / k_{m}^{2}}}{\sqrt{k_{\perp}^{2}-k_{x}^{2}}} \\
& =\frac{1}{2 k_{x}} \cot ^{-1}\left(\frac{k_{x}}{k_{m}}\right)+\frac{1}{4 k_{m}} \ln \left(\frac{4 k_{\perp}^{*^{2}}}{k_{m}^{2}+k_{x}^{2}}\right)
\end{aligned}
$$

and

$I_{b} \simeq \int_{k_{\perp}^{*}}^{\infty} \frac{d k_{\perp}}{2 k_{m} k_{\perp}} \frac{\sin ^{2}\left(k_{\perp} / k_{\mathrm{R}}\right)}{\left(k_{\perp} / k_{\mathrm{R}}\right)^{2}} \simeq-\frac{1}{2 k_{m}} \ln \left(\frac{2 e^{3 / 2-\gamma} k_{\perp}^{*}}{k_{\mathrm{R}}}\right)$

Adding Eqs. (46) and (47) we have

$I \simeq \frac{1}{2 k_{m}}\left\{\frac{k_{m}}{k_{x}} \cot ^{-1}\left(\frac{k_{x}}{k_{m}}\right)+\ln \left[\frac{e^{3 / 2-\gamma} k_{\mathrm{R}} / k_{m}}{\sqrt{1+\left(k_{x} / k_{m}\right)^{2}}}\right]\right\}$

Plugging Eq. (48) in Eq. (44), and calling $\bar{k} \equiv k_{x} / k_{\mathrm{M}}$, with $\bar{k}_{m} \equiv k_{m} / k_{\mathrm{M}}$ and $\bar{k}_{\text {int }} \equiv k_{\text {int }} / k_{\mathrm{M}} \ll \bar{k}_{m}$, we can write

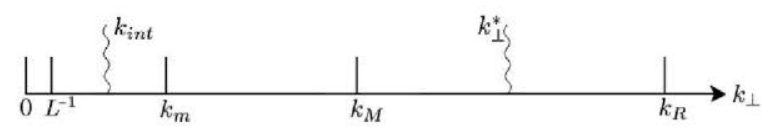

Fig. 3. Characteristic regions for $k_{\perp} .1 / L \ll k_{\text {int }} \ll k_{m} \ll k_{\mathrm{M}} \ll k_{\perp}^{*} \ll k_{\mathrm{R}}$. 


$$
\begin{aligned}
\mathscr{Z}_{A 1} \approx & \frac{1}{2} \int_{\bar{k}_{\text {int }}}^{1} \frac{d \bar{k}}{\bar{k}} \sqrt{1-\bar{k}^{2}} \cot ^{-1}\left(\frac{\bar{k}}{\bar{k}_{m}}\right)+\frac{1}{2 \bar{k}_{m}} \\
& \times \int_{0}^{1} d \bar{k} \sqrt{1-\bar{k}^{2}} \ln \left[\frac{k_{\mathrm{R}} e^{3 / 2-\gamma}}{k_{m} \sqrt{1+\left(\bar{k} / \bar{k}_{m}\right)^{2}}}\right]
\end{aligned}
$$

where we included the range $0 \leqslant \bar{k} \leqslant \bar{k}_{\text {int }}$ in the second integral, where it makes a negligible contribution of order $\bar{k}_{\text {int }}$. Evaluating the $\bar{k}$ integrals yields

$$
\mathscr{Z}_{A 1} \simeq \frac{\pi}{4}\left\{\ln \left[\frac{2 \bar{k}_{m}}{\bar{k}_{\text {int }}\left(1+\sqrt{1+\bar{k}_{m}^{2}}\right)}\right]+\frac{1}{2 \bar{k}_{m}} \ln \left[\frac{2 \bar{k}_{m} e^{2-\gamma} k_{\mathrm{R}}}{k_{m}\left(1+\bar{k}_{m}\right)}\right]\right\}
$$

For region $2\left(0 \leqslant k_{x} \leqslant k_{\text {int }}\right)$, introducing $\kappa \equiv k_{y} / k_{x}$ and using $k_{x} \ll k_{\mathrm{M}}$, we find

$$
\mathscr{Z}_{A 2} \approx \int_{0}^{\bar{k}_{\text {int }}} \frac{d \bar{k}}{\bar{k}} \int_{0}^{\infty} \frac{d \kappa}{1+\kappa^{2}} \sin ^{2}\left(\frac{\bar{k} \Lambda \kappa}{2}\right)
$$

where $\Lambda \equiv k_{\mathrm{M}} L \sin \varphi$. We readily find

$\int_{0}^{\infty} \frac{d \kappa}{1+\kappa^{2}} \sin ^{2}\left(\frac{\bar{k} \Lambda \kappa}{2}\right)=\frac{\pi}{4}\left(1-e^{-\bar{k} \Lambda}\right)$

With $1 / L k_{\mathrm{M}} \ll \bar{k}_{\text {int }}$, the resultant integral is

$\mathscr{Z}_{A 2}=\frac{\pi}{4} \int_{0}^{\bar{k}_{\text {int }}} \frac{d \bar{k}}{\bar{k}}\left(1-e^{-\bar{k} A}\right) \simeq \frac{\pi}{4}\left[\ln \left(\Lambda e^{\gamma}\right)+\ln \left(\bar{k}_{\text {int }}\right)\right]$

Adding Eqs. (50) and (53) we have

$\mathscr{Z}_{\mathrm{A}} \simeq \frac{\pi}{4}\left[\ln \left(\bar{k}_{m} \Lambda e^{\gamma}\right)+\frac{1}{2 \bar{k}_{m}} \ln \left(\frac{2 \bar{k}_{m} e^{2-\gamma} k_{\mathrm{R}}}{k_{m}\left(1+\bar{k}_{m}\right)}\right)\right]$

To obtain the Alfven impedance radiation for a spinning tether, we now averaging $\mathscr{Z}_{\mathrm{A}}$ over one revolution. Using Eq. (54) in Eq. (40) and $\Lambda \propto \sin \varphi$, yields

$\frac{2}{\pi} \int_{0}^{\pi / 2} d \varphi \ln \left(k_{\mathrm{M}} L \sin \varphi\right)=\ln \left(\frac{k_{\mathrm{M}} L}{2}\right)$

the averaged impedance has the final compact form

$Z_{\mathrm{A}} \approx \frac{2 V_{\mathrm{A}}}{c^{2}}\left\{\ln \left[\frac{e^{\gamma} L \omega_{\mathrm{pe}}}{2 c}\right]+\frac{1}{2} \frac{\Omega_{i} c}{V_{\mathrm{pl}} \omega_{\mathrm{pe}}} \ln \left[\frac{2 V_{\mathrm{pl}} e^{2-\gamma}}{R \Omega_{i}}\right]\right\}$

To compare the impedance above with the equation (19') for the impedance at LEO in Sanmartín and Martínez-Sánchez (1995), which reads

$Z_{\mathrm{A}}=\frac{2 V_{\mathrm{A}}}{c^{2}} \ln \left(\frac{2 e^{\gamma-1} L \Omega_{i}}{V_{\text {orb }}}\right)$

note first that in Eq. (56), the ratio $\Omega_{i} c / V_{\mathrm{pl}} \omega_{\mathrm{pe}} \simeq$ $73.2 \mathrm{~m} / 14.2 \mathrm{~m} \simeq 5.2$ from Eq. (42) is moderately large. In LEO, however, that ratio is two orders of magnitude smaller because $\Omega_{i}$ and $N_{\mathrm{e}}$ are about 10 times smaller and $10^{2}$ times larger, respectively; this made the $R$-logarithm term, which was actually ignored in Sanmartín and Martínez-
Sánchez (1995), indeed negligible. Secondly, moving from the Jovian to the LEO case, i.e., moving from condition $\omega_{\mathrm{pe}} / c \ll \Omega_{i} / V_{\mathrm{pl}}$ to $\Omega_{i} / V_{\mathrm{pl}} \ll \omega_{\mathrm{pe}} / c$, the length ratio entering the first logarithm for $Z_{\mathrm{A}}$ in Eq. (56) would change from $L \omega_{\mathrm{pe}} / c$ to $L \Omega_{i} / V_{\mathrm{pl}}$. In addition, in Eq. (57) for LEO, the perpendicular relative velocity (which is then just about the orbital velocity $V_{\text {orb }}$ ) does figure instead of $V_{\mathrm{pl}}$ because of the differences in orbital geometry, i.e., equatorial instead of polar (at $\lambda=0$ ), which determines the velocity for the Doppler relation, Eq. (2). Finally, the factor $2 / e$ in the logarithm of Eq. (57), i.e., the small $\ln (2 / e)$ contribution to the large logarithm in $Z_{\mathrm{A}}$, which is missing from Eq. (56), was lost in the approximation leading to $\mathrm{Eq}$. (51); further, the $1 / 2$ factor in Eq. (56) is due to the spinning tether, not used in LEO.

\section{The radiation impedance at the polar caps}

In the polar Jovian case, we have $\omega=k_{y} V_{\text {orb }} / \sqrt{2}<\Omega_{i}$, $k_{y}$ thus being less than a maximum value $k_{\mathrm{M}}=\sqrt{2} \Omega_{i} / V_{\text {orb }}$. The integrand in Eq. (36) is written here as

$Z_{\mathrm{A}}=\frac{8 V_{\mathrm{A}}}{\pi c^{2} \sqrt{1+\left(V_{\mathrm{A}} / c\right)^{2}}} \int_{0}^{\pi / 2} \frac{d \varphi}{\pi / 2} \mathscr{Z}_{\mathrm{A}}$

where

$$
\begin{aligned}
\mathscr{Z}_{\mathrm{A}}= & \int_{0}^{k_{\mathrm{M}}} d k_{y} \frac{\sqrt{1-k_{y}^{2} / k_{\mathrm{M}}^{2}} \sin ^{2}\left(\frac{1}{2} k_{y} L \sin \varphi\right)}{\sqrt{1-\widetilde{V}_{\mathrm{A}}^{2} k_{y}^{2} / k_{\mathrm{M}}^{2}}} \\
& \times \int_{0}^{\infty} \frac{d k_{x}}{k_{\perp}^{2}} \sqrt{1+\frac{k_{\perp}^{2}}{k_{m}^{2}}} \frac{\sin ^{2}\left(k_{\perp} / k_{\mathrm{R}}\right)}{\left(k_{\perp} / k_{\mathrm{R}}\right)^{2}}
\end{aligned}
$$

with

$k_{m} \equiv \frac{\omega_{\mathrm{pe}}}{c} \simeq \frac{1}{2.37 \mathrm{~km}}$

$k_{\mathrm{M}} \equiv \sqrt{2} \frac{\Omega_{i}}{V_{\text {orb }}} \simeq \frac{1}{118.24 \mathrm{~m}}$

$\widetilde{V}_{\mathrm{A}}^{2} \equiv \frac{V_{\mathrm{A}}^{2}}{V_{\mathrm{A}}^{2}+c^{2}} \simeq 0.18$

Using the same procedure as in the equatorial case, we change the integration from $k_{x}$ to $k_{\perp}=\sqrt{k_{y}^{2}+k_{x}^{2}}$ at fixed $k_{y}$, and evaluate first the $k_{\perp}$ integral, which reads

$$
\mathscr{Z}_{\mathrm{A}} \simeq \int_{0}^{k_{\mathrm{M}}} d k_{y} \frac{\sqrt{1-k_{y}^{2} / k_{\mathrm{M}}^{2}}}{\sqrt{1-\widetilde{V}_{\mathrm{A}}^{2} k_{y}^{2} / k_{\mathrm{M}}^{2}}} \sin ^{2}\left(k_{y} \frac{L}{2} \sin \varphi\right) \cdot I\left(k_{y}\right)
$$

where

$I\left(k_{y}\right) \simeq \frac{1}{k_{m}}\left[\frac{k_{m}}{k_{y}} \cot ^{-1}\left(\frac{k_{y}}{k_{m}}\right)+\ln \left\{\frac{k_{\mathrm{R}} / k_{m} e^{3 / 2-\gamma}}{\sqrt{1+\left(k_{y} / k_{m}\right)^{2}}}\right\}\right]$ 
where $I\left(k_{y}\right)$ is just $2 I$ as given in Eq. (48), with a $k_{x} \rightarrow k_{y}$ change.

Calling $\mathscr{Z}_{\mathrm{AI}}$ and $\mathscr{Z}_{\mathrm{AII}}$ the contributions of the first and second terms in the bracket of Eq. (64) to the impedance in Eq. (63), we readily find

$\mathscr{Z}_{\mathrm{AII}} \simeq \frac{k_{\mathrm{M}}}{2 k_{m}} \int_{0}^{1} d \bar{k} \frac{\sqrt{1-\bar{k}^{2}}}{\sqrt{1-\widetilde{V}_{\mathrm{A}}^{2} \bar{k}^{2}}} \ln \left\{\frac{k_{\mathrm{R}} / k_{m} e^{3 / 2-\gamma}}{\sqrt{1+\left(k_{\mathrm{M}} / k_{m}\right)^{2} \bar{k}^{2}}}\right\}$

where we set $\sin ^{2}\left(\frac{1}{2} k_{y} L \sin \varphi\right) \simeq 1 / 2$ and wrote $k_{y} / k_{\mathrm{M}} \equiv \bar{k}$. With the approximation $1 / \sqrt{1-\widetilde{V}_{\mathrm{A}}^{2} \bar{k}^{2}} \approx 1+\widetilde{V}_{\mathrm{A}}^{2} \bar{k}^{2} / 2$, Eq. (65) can be analytically determined,

$\mathscr{Z}_{\mathrm{AII}} \simeq \frac{\pi k_{\mathrm{M}}}{8 k_{m}}\left[\ln \left(\frac{2 e^{2-\gamma}}{R k_{\mathrm{M}}}\right) \cdot\left\{1+\frac{\widetilde{V}_{\mathrm{A}}^{2}}{8}\right\}-\frac{3 \widetilde{V}_{\mathrm{A}}^{2}}{32}\right]$

We now check the approximation above, by computing Eq. (65) numerically, using typical values expressed in Eqs. (60)-(62) and $k_{\mathrm{R}}=0.5 \mathrm{~m}^{-1}$. This shows an error less than $2 \%$. Actually, the last term in the square bracket of Eq. (66) represents a correction of $0.3 \%$, and will be dropped.

For $\mathscr{Z}_{\mathrm{AI}}$, where small $k_{y} / k_{\mathrm{M}}$ values are dominant, we must retain the full square sine factor. Defining $\tilde{k} \equiv \frac{1}{2} k_{y} L \sin \varphi$, we have

$\mathscr{Z}_{\mathrm{AI}}\left(\tilde{k}_{m}\right) \simeq \int_{0}^{\infty} \frac{d \tilde{k}}{\tilde{k}} \sin ^{2} \tilde{k} \cdot \cot ^{-1}\left[\frac{\tilde{k}}{\tilde{k}_{m}}\right]$

As this integral converges rapidly at large $\tilde{k}$ values, we set $\tilde{k}_{\mathrm{M}} \simeq \infty$, and approximated the square root factors in Eq. (63) by unity. From Eq. (67) and using $\mathscr{Z}_{\mathrm{AI}}(0)=0$, we find

$\frac{d \mathscr{Z}_{\mathrm{AI}}}{d \tilde{k}_{m}}=\int_{0}^{\infty} d \tilde{k} \frac{\sin ^{2} \tilde{k}}{\tilde{k}_{m}^{2}+\tilde{k}^{2}}=\frac{\pi}{4 \tilde{k}_{m}}\left(1-e^{-2 \tilde{k}_{m}}\right)$

$\mathscr{Z}_{\mathrm{AI}}=\frac{\pi}{4}\left\{\ln \left(2 \tilde{k}_{m}\right)+\gamma+E_{1}\left(2 \tilde{k}_{m}\right)\right\} \simeq \frac{\pi}{4} \ln \left(L e^{\gamma} k_{m} \sin \varphi\right)$

where we used an approximation of the exponential integral $E_{1}$ roughly valid for its argument above unity.

Introducing Eqs. (66) and (69) in Eq. (58), and averaging over a spin period, we finally find

$$
\begin{aligned}
Z_{\mathrm{A}} \simeq & \frac{2 V_{\mathrm{A}}}{c^{2} \sqrt{1+V_{\mathrm{A}}^{2} / c^{2}}}\left\{\ln \left(\frac{L e^{\gamma} \omega_{\mathrm{pe}}}{2 c}\right) .\right. \\
& \left.+\frac{\Omega_{i} c}{\sqrt{2} \omega_{\mathrm{pe}} V_{\text {orb }}}\left(1+\frac{V_{\mathrm{A}}^{2} / 8}{V_{\mathrm{A}}^{2}+c^{2}}\right) \ln \left(\frac{\sqrt{2} V_{\mathrm{orb}} e^{2-\gamma}}{R \Omega_{i}}\right)\right\}
\end{aligned}
$$

One would recover Eq. (56) by first setting $V_{\mathrm{A}}^{2} / c^{2}$ small and replacing $V_{\text {orb }} / \sqrt{2}$ with $V_{\mathrm{pl}}$.

The main impedance differences among Jovian equatorial and polar, and LEO cases, is shown in a graphical form in Fig. 4.

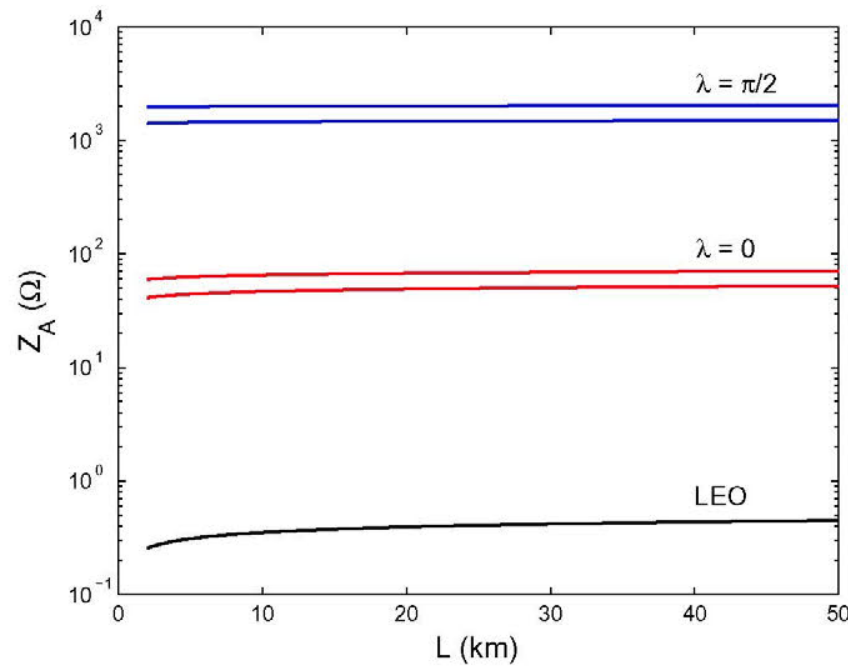

Fig. 4. Impedance versus tether length for the LEO, and Jovian equatorial and polar cases. We use values given in Table 2 for $R=0.5$ and $4 \mathrm{~m}$ for the upper and lower curves, respectively.

\section{Conclusions}

The Alfven radiation impedance $Z_{\mathrm{A}}$ for an insulated conductive tether in a Juno-type orbit has been determined. Plasma density, $N_{\mathrm{e}}$, and magnetic field $B$ are here much smaller and much larger than in LEO, respectively. The Alfven velocity, which varies as $V_{\mathrm{A}} \propto B / \sqrt{N_{\mathrm{e}}}$, is thus much larger for Juno. Since the Alfven impedance is basically proportional to $V_{\mathrm{A}}$, it will be much larger too. The highest Alfven velocity, occurring at the polar caps, gives an impedance radiation three orders of magnitude greater than in LEO.

Juno's low density and high magnetic field values modify the Alfven power spectrum found at LEO, too. For Juno, the condition $c / \omega_{\mathrm{pe}} \gg V_{\text {rel } \perp} / \Omega_{i}$ holds whereas the opposite applies to LEO, $V_{\text {rel } \perp}$ being the component of the orbit-to-plasma relative velocity that is perpendicular to the magnetic field. There are here two contributions to the Alfven impedance, involving logarithms of two large length ratios, $L \omega_{\mathrm{pe}} / c$ and $V_{\text {rel } \perp} / R \Omega_{i}$ (aside of numerical

Table 2

Summary of plasma and velocity characteristic values. We use a value $r_{\mathrm{p}}=1.09 R_{\mathrm{J}}$ for the perijove, and typical Earth plasma parameters in LEO. As later shown, a limiting wave number, $\Omega_{i} / V_{\text {rel } \perp}$, is $\Omega_{i} / V_{\mathrm{pl}}$ at $\lambda=0$ and $\sqrt{2} \Omega_{i} / V_{\text {orb }}$ at the polar caps. The relevant length scale $c / \omega_{\mathrm{pe}}$ takes quite different values in LEO and Jupiter.

\begin{tabular}{llll}
\hline Parameters & Earth & Jupiter $(\lambda=0)$ & Jupiter $(\lambda=\pi / 2)$ \\
\hline$V_{\text {orb }}(\mathrm{km} / \mathrm{s})$ & 7.5 & 56.9 & 40.2 \\
$V_{\mathrm{pl}}(\mathrm{km} / \mathrm{s})$ & - & 13.9 & 0 \\
$V_{\mathrm{A}}(\mathrm{km} / \mathrm{s})$ & 300 & $17.3 \times 10^{3}$ & $14.6 \times 10^{4}$ \\
$\Omega_{i}\left(\mathrm{~s}^{-1}\right)$ & $2.0 \times 10^{2}$ & $9.4 \times 10^{2}$ & $2.3 \times 10^{2}$ \\
$\Omega_{\mathrm{e}}\left(\mathrm{s}^{-1}\right)$ & $5.9 \times 10^{6}$ & $5.6 \times 10^{7}$ & $1.4 \times 10^{7}$ \\
$\omega_{\mathrm{pi}}\left(\mathrm{s}^{-1}\right)$ & $2.0 \times 10^{5}$ & $1.6 \times 10^{4}$ & $4.8 \times 10^{2}$ \\
$\omega_{\mathrm{pe}}\left(\mathrm{s}^{-1}\right)$ & $3.4 \times 10^{7}$ & $3.9 \times 10^{6}$ & $1.2 \times 10^{5}$ \\
$c / \omega_{\mathrm{pe}}(\mathrm{m})$ & 8.8 & 73.2 & $2.37 \cdot 10^{3}$ \\
$V_{\text {rel } \perp} / \Omega_{i}(\mathrm{~m})$ & 35.6 & 14.2 & 118.24 \\
\hline
\end{tabular}


factors of order unity). In LEO, $Z_{\mathrm{A}}$ involved just the logarithm of $L \Omega_{i} / V_{\text {rel } \perp \text {. }}$.

Fully determining the Alfven impedance for Juno required a model for the contactor radius $R$. Whether the radius of a thin sheath for a passive contactor, or a contactor cloud radius for an active one, a model $R \sim \sqrt{I_{\mathrm{s}} / 4 \pi j_{\mathrm{th}}}$ had been suggested in the literature for FMS radiation in LEO. Typically, a radius $R$ around one meter can be expected. Typical lengths $L \sim 20 \mathrm{~km}$, and $R \sim 2 \mathrm{~m}$ make for large values of the ratio $L \omega_{\mathrm{pe}} / c$, and of ratios $V_{\mathrm{pl}} / R \Omega_{i}$ and $V_{\text {orb }} / R \Omega_{i}$ at $\lambda=0$ and $\lambda=\pi / 2$, respectively. This means that the impedance can be found to just logarithmic accuracy, a factor of 2 , say, in either $L$ or $R$ changing weakly the logarithm values. Actually, ion thermal effects might enter the $\ln \left(V_{\text {rel } \perp} / R \Omega_{i}\right)$ term at the perijove of the Juno orbit, where $V_{\text {thi }}$ is close to $V_{\text {rel } \perp}$. Again, this would result in just logarithmically accuracy for the $Z_{\mathrm{A}}$ dependence on $R$.

The analysis proposed in this paper could be extended to a fully bare tether by properly changing the model for plasma-tether current contact, i.e., the source divergence in Eq. (37). The first term in that equation corresponds to the cathodic contactor, and would be kept as it is, whereas the second term should be modified to accommodate collection by the positively biased segment of the tether. This makes for a more complex dimensionless function $g(\mathbf{k})$ in Eq. (38) to use in Eq. (36). However, the computation of the radiation impedance for a bare tether in a Juno-like mission goes beyond the scope of the present paper and will be analyzed in future work; for the LEO case, the radiation impedance for a bare tether had a similar expression and was comparable to the impedance of an insulated tether (Sanmartín and Martínez-Sánchez, 1995).

\section{Acknowledgments}

Part of this work was supported by the Ministry of Science and Innovation of Spain (BES-2009-013319 FPI Grant), and the Ministry of Science and Technology of
Spain under Grant No. ESP2004-01511. We acknowledge helpful comments and criticism by the referees.

\section{References}

Akhiezer, A.I., Polovin, R.V., Sitenko, A.G., Stepanov, K.N.. Plasma Electrodynamics, vol. 1. Pergamon Press, New York, 1975.

Bagenal, F. The magnetosphere of Jupiter: coupling the equator to the poles. J. Atmos. Sol-Terr. Phys. 69, 387-402, 2007.

Barnett, A., Olbert, S. Radiation of plasma waves by a conducting body moving through magnetized plasma. J. Geophys. Res. 91 (10), 10117 10135, 1986.

Bombardelli, C., Lorenzini, E.C., Sanmartín, J.R. Jupiter power generation with electrodynamic tethers at constant orbital energy. J. Propul. Power 25 (2), 415-423, 2009.

Divine, N.T., Garrett, H.B. Charged particle distributions in Jupiter's magnetosphere. J. Geophys. Res. 88, 6889-6903, 1983.

Dobrowolny, M., Veltri, P. MHD power radiated by a large conductor in motion through a magnetoplasma. Nuovo Cimento Soc. Ital. Fis. C 9, $27-38,1986$

Donohue, D.J., Neubert, T., Banks, P.M. Estimating radiated power from a conducting thethered satellite system. J. Geophys. Res. 96, 21245 21253, 1991.

Drell, S.D., Foley, H.M., Ruderman, M.A. Drag and propulsion of large satellites in the ionosphere: an Alfven propulsion engine in space. J. Geophys. Res. 70 (13), 3131-3145, 1965.

Estes, R.D. Alfven waves from an electrodynamic tethered satellite system. J. Geophys. Res. 93, 945-956, 1988.

Hastings, D.E., Wang, J. The radiation impedance of an electrodynamic tether with end connectors. J. Geophys. Res. Lett. 14, 519-522, 1987.

Hastings, D.E., Wang, J. Induced emission of radiation from a large space-station-like structure in the ionosphere. AIAA J. 27, 438-445, 1989.

Matousek, S. The Juno New Frontiers mission. Acta Astronaut. 61, $923-$ 939, 2007.

National Academy of Sciences. Radioisotope Power Systems: An imperative for Maintaining US Leadership in Space Exploration. Available from: <http://books.nap.edu/catalog/12653.html>, 2009.

Sanmartín, J.R., Martínez-Sánchez, M. The radiation impedance of orbiting conductors. J. Geophys. Res. 100, 1677-1686, 1995.

Sanmartín, J.R., Charro, M., Lorenzini, E.C., Garrett, H.B., Bombardelli, C., Bramanti, C. Electrodynamic tether at Jupiter-I: capture operation and constraints. IEEE Trans. Plasma Sci. 36 (5), 2450-2458, 2008.

Sanmartín, J.R., Charro, M., Lorenzini, E.C., Garrett, H.B., Bombardelli, C., Bramanti, C. Electrodynamic tether at Jupiter-II: fast Moon tour after capture. IEEE Trans. Plasma Sci. 37 (4), 620-626, 2009. 